\title{
Aptamer-Functionalized Quercetin Thermosensitive Liposomes for Targeting Drug Delivery and Antitumor Therapy
}

Jian Li ( $\sim$ Lijianbio@ysu.edu.cn )

Yanshan University

Yanting Gao

Yanshan University

Shihe Liu

Yanshan University

Jiahui Cai

Yanshan University

Qing Zhang

Yanshan University

Kun Li

Yanshan University

Zhiwei Liu

Yanshan University

Ming Shi

Yanshan University

Jidong Wang

Yanshan University

Hongxia Cui

Yanshan University

\section{Research Article}

Keywords: quercetin, thermosensitive liposomes, thermotherapy, AS1411 aptamer, tumor therapy

Posted Date: February 8th, 2022

DOI: https://doi.org/10.21203/rs.3.rs-1331144/v1

License: (c) (i) This work is licensed under a Creative Commons Attribution 4.0 International License.

Read Full License 


\section{Abstract}

Chemo-thermotherapy, as a promising cancer combination therapy strategy, has attracted widespread attention. In this study, a novel aptamer functionalized thermosensitive liposomes encapsulating hydrophobic drug quercetin were fabricated as an efficient drug delivery system. This aptamerfunctionalized quercetin thermosensitive liposomes (AQTSL) combined the merits of high-loading yield, sustained drug release, long-term circulation in the body of PEGylated liposomes, passive targeting of 100-200 nm nanoparticles, active targeting and improved internalization effects offered by AS1411 aptamer, and quercetin release controlled by temperature-response and thermotherapy. In addition, AQTSL tail vein injection combined with $42^{\circ} \mathrm{C}$ administration inhibited the tumor growth significantly compared with the saline administration $(\mathrm{p}<0.01)$, and the inhibition rate reached $75 \%$. Furthermore, AQTSL combined with $42^{\circ} \mathrm{C}$ administration also slowed down the tumor growth significantly compared with QTSL combined with $42^{\circ} \mathrm{C}$ administration $(\mathrm{p}<0.05)$, which further confirmed that AS1411 decoration increased the active targeting and internalization effects of the drug delivery system, and AS1411 aptamer itself might also contribute to the tumor inhibition process. These data indicated that AQTSL is a potential carrier candidate for different hydrophobic drug tumor targeting delivery and this kind of targeted drug delivery system combined with temperature response drug release mode is expected to achieve an ideal tumor therapy effect.

\section{Introduction}

During the therapy of malignant tumors, the non-tumor targeted transport of chemotherapy drugs shows serious impacts on normal tissue cells, reduces the dose of drugs reaching tumor tissue, which will compromise the therapeutic effects of drugs [1]. Therefore, the development of new drugs and new treatment options is a challenge for malignant tumors therapy. Drugs can be encapsulated in the nanoparticles for the regulation of their release rate, change of their distribution in vivo, and the improvement of their bioavailability. In addition, compared with traditional medicine, nano-drug delivery systems possess the following advantages: 1) The ideal size range of the nano-carriers is $100-200 \mathrm{~nm}$, for it can pass through the capillary near the tumor and accumulate at the tumor tissues through the enhanced permeability and retention (EPR) effect and can also avoid the phagocytosis by the reticuloendothelial system. 2) They often have many cavities or special surfaces, which can load hydrophobic chemotherapy drugs with improved drug solubility and reduced side effects. 3) They often have the characteristics of slow-release and controlled release for the loaded drugs, which provide prolonged circulation time of drugs in the body, up-regulated the local drug concentration in tumor tissues, and improve the therapeutic effect of drugs. And 4) The surface of nano-carriers is easy modified by targeting molecules, which increase the targeting during the transportation and further alleviate the toxic effects of chemotherapy drugs [2].

The following steps are involved in drug delivery systems in vivo: 1) efficient drug loading or encapsulation; 2) drug stability in vivo circulation; 3) specific accumulation in tumor tissues; 4) efficient tumor cell membrane transportation; 5) successful lysosomal escape; 6) responsive drug release at target 
sites, 7) effective nuclear transport (when the target locates in the nucleus). Only when these steps are completed, can the precise release of the payload on the drug delivery system at specific target sites be achieved.

Liposomes are dominant nano-drug delivery platforms due to their capability of loading hydrophilic drugs at high encapsulation efficiency using their hydrophilic core cavity and carrying of hydrophobic drugs exploiting the hydrophobic cavity between phospholipid bilayer membranes simultaneously. Nanoliposome drug delivery system also possesses ideal pharmacokinetic characteristics, good biocompatibility, and easy surface modification. Furthermore, to reduce the clearance of reticuloendothelial system and increase the circulation time of drugs in vivo, liposomes can also be further encapsulated by inert and hydrophobic polyethylene glycol (PEG) [3].

The targeting ligand modified targeted drug delivery system (DDS) is anticipated to achieve better treatment effect because it augments the selectivity and local dose of drugs to tumor cells [4]. Aptamer is a single-stranded DNA, RNA, or modified nucleic acid screened by systematic evolution of ligands by exponential enrichment (SELEX) method [5] which specifically binds to abundant targets such as small molecules, proteins, cells, and even tissues by its unique secondary or tertiary structure. Therefore, aptamers with high affinity to the specific proteins on tumor cell surface can be used as ligands for targeting DDS.

Nucleolin, which locates in the nucleus of normal cells abundantly and over-expressed on the membrane of tumor cells [6], is involved in the transport of molecules from the cell surface to nucleus, cell proliferation, and DNA replication [7, 8]. Nucleolin aptamer, AS1411, is a guanosine-rich oligonucleotide and showed a high affinity with nucleolin [9]. Moreover, the aptamer itself has anti-proliferation and cytotoxicity to tumor cells, and then induces a nonapoptotic cell death - methuosis [10,11]. Our previous study also showed that HeLa cells showed a high affinity with AS1411, much higher than another aptamer EpCAM. Hence, we chose AS1411 as targeting moiety and expected them to guide nano-carriers effectively targeting HeLa cells overexpressing nucleolin on their cell surface $[12,13]$.

Therefore, we prepared thermo-sensitive quercetin liposomes (AQTSL) by improving the previous synthesis method and constructed a temperature-responsive and actively targeted drug delivery system (Scheme 1A). Structurally, this nano-carrier is composed of 1) thermosensitive liposomes, which can improve the quercetin water solubility and achieve the temperature-controlled drug release; 2 ) an anticancer drug quercetin which can affect the proliferation, metastasis, apoptosis and metabolism of tumor cells through multiple signaling pathways; 3 ) a hydrophilic polymer stealth layer outside the lipid shell made of pegylated phospholipids 1,2-distearoyl-sn-glycero-3-phosphoethanolamine-N-carboxy (polyethyleneglycol) 2000 (DSPE-PEG2000-COOH), which improves the stability of liposomes and shows a reduced tendency to leak drugs while in circulation; 4) anti-nucleolin aptamers AS1411, which can gain entry for the carriers into the cells when the nucleolin receptors shuttle from the plasma membrane into the cytoplasm and nucleus on one hand, and on the other hand, AS1411 aptamer itself can also act as an aptamer drug and exhibit antiproliferative activity against tumor cells $[14,15]$. 
Due to the passive targeting of the liposomes and active targeting of the aptamer AS1411, the anti-tumor drug quercetin can be efficiently aggregated at the tumor site. Meanwhile, the increase of external temperature not only causes the liposomes to undergo a phase change and release quercetin for active and controlled drug release but also induces the tumor cells apoptosis owing to tumor cells are more sensitive to $42^{\circ} \mathrm{C}$ temperature than the normal cells. The natural product quercetin inhibits the proliferation of malignant cells by selectively inducing the degradation of the oncogene Ras protein, blocking protein expression in the G2/M phase of the cell cycle, and inducing endogenous pathwaymediated apoptosis involving the cellular caspase 9 and 3 proteins. Together with AS1411, being a small molecule nucleic acid drug is expected to exhibit ideal therapeutic effects on cancers (Scheme 1B).

\section{Materials And Methods}

\subsection{Materials and kits}

Quercetin (Que, purity $\geq 98 \%$ ) was obtained from North China Pharmaceutical Co., Ltd.

Dipalmitoylphosphatidylcholine (DPPC) \mono-stearoyl-phosphatidyl-choline (MSPC), and DSPE-PEG $2000^{-}$ $\mathrm{COOH}$ were purchased from Shanghai AVT Pharmaceutical Technology Co., Ltd. (Shanghai, China).

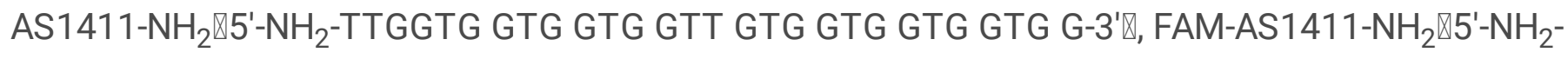

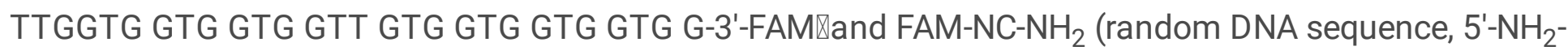
CCT CCT TCCTTC AAA ACA ACC AAC CACC-3'-FAM were obtained from Sangon Biotech. Co., Ltd. (Shanghai, China). sulforhodamine B (SRB) staining kits and Annexin V-FITC/PI Apoptosis Detection Kits were obtained from Beyotime Biotech. Co., Ltd. (Beijing, China).

\subsection{Cell lines and animals}

The human renal epithelial 293T cell line and human cervical cancer HeLa cell line was kindly gifted by Professor Chang at Tsinghua University. Mouse cervical cancer U14 cell line was provided by Tongpai (Shanghai) Biotechnology Co., Ltd. All cells were cultured in DMEM media (containing 10\% FBS and 1\% penicillin-streptomycin) at $37^{\circ} \mathrm{C}$ in an incubator supplied with $5 \% \mathrm{CO}_{2}$ (Thermo Scientific, Waltham, MA).

Kunming mice (female, 20 \pm 2 g, Lot No. 1100111911024294, License No. SCXK(Beijing) 2016-0011) were got from Beijing River Laboratory Animal Technology Co., Ltd. All the animals were raised under a standard SPF environmental conditions (12-hour-light/-dark cycle, $22 \pm 2^{\circ} \mathrm{C}$ room temperature) with free access to food and water. All procedures with the experimental mice shall comply with the National Institutes of Health Guide for the Care and Use of Laboratory Animals strictly and was approved by the Committee of Animal Care and Use in Yanshan University of China (Ethics number: YD2019012)

\subsection{Liposomes preparation}

AQTSL was prepared by thin-film hydration [16]. Briefly, $100 \mu \mathrm{L}$ of DSPE-PEG2000-COOH $(0.15 \mathrm{mg} / \mathrm{mL})$ and $100 \mu \mathrm{L}$ activation solution (EDC/NHS) were mixed and sonicated in an ice-water bath for $30 \mathrm{~min}$ for further aptamer linking, then the AS1411- $\mathrm{NH}_{2}(5 \mu \mathrm{M})$ was added and sonicated in a water bath for $1 \mathrm{~h}$, 
followed by reaction for $12 \mathrm{~h}$ to get AS1411-NH2-DSPE-PEG2000. Next, the products were separated with two kinds of ultrafiltration tubes ( $\mathrm{MWCO}=4 \mathrm{KD}$ and $\mathrm{MWCO}=10 \mathrm{KD}$, respectively) to remove the unreacted DSPE-PEG2000- $\mathrm{COOH}$ and AS1411- $\mathrm{NH}_{2}$. The upper layer of the second ultrafiltration tube was shaken by vertexing on an oscillator, and the liquid in the tube was transferred to a new EP tube and stored at $4^{\circ} \mathrm{C}$.

DPPC, MSPC, and quercetin were weighed with a mass ratio of 9:1:1 and dissolved in a mixture of methanol and dichloromethane with a volume ratio of 2:1. The liquid mixture above was placed in a flask, sonicated for $2 \mathrm{~min}$ to dissolve the substances completely, followed by evaporating using a rotary evaporator. Next, the dried lipid film was hydrated with the AS1411-DSPE-PEG2000 coupling reagent to obtain the final lipid. Next, AQTSL were prepared by passing through $0.45 \mu \mathrm{m}, 0.2 \mu \mathrm{m}$, and $0.1 \mu \mathrm{m}$ membranes sequentially and extruding the resulting liposomes through $0.1 \mu \mathrm{m}$ polycarbonate filter membrane 60 times at $60^{\circ} \mathrm{C}$ using an extruder to make the particle size more uniform. Finally, the liposomes were packed into $14000 \mathrm{KD}$ dialysis bags and dialyzed in neutral PBS solution for 2 days to remove the free quercetin from the system.

Thermosensitive liposomes (TSL), quercetin thermosensitive liposomes (QTSL), negative control (freedom DNA sequence) modified quercetin thermosensitive liposomes (NCQTSL), FAM decorated NCQTSL (FAM-NCQTSL), and FAM decorated AQTSL (FAM-AQTSL) were prepared according to the methods above with minor revisions respectively.

\subsection{Characterizing of nanoparticles}

A transmission electron microscopy (TEM, HT-7700, Hitachi, Japan.) was applied to observe the morphology of AQTSL, and a Malvern Zetasizer (Nano ZS, Malvern Instruments Ltd., U.K.) was used to measure the particle size and polydispersity index (PDI) of liposomes.

Measurement of the phase transition temperature was performed using a differential scanning calorimetry (DSC/DTA STA 449 F5 Jupiter, Netzsch, German). The samples were scanned with an average heating rate of $10^{\circ} \mathrm{C} / \mathrm{min}$ from $10^{\circ} \mathrm{C}$ to $70^{\circ} \mathrm{C}$, and the nitrogen flow rate was $20 \sim 30$ $\mathrm{mL} / \min [17]$.

2.5. Drug encapsulation efficiency (EE) and drug release study of AQTSL in vitro

Firstly, the standard curve for quercetin of gradient concentrations vs their absorbances at $370 \mathrm{~nm}$ was drawn. Next, the obtained QTSL or AQTSL (without dialysis) was then placed in an ultrafiltration tube (10 $\mathrm{KD}$, Millipore) and followed by centrifuging to separate the non-encapsulated quercetin from QTSL or AQTSL (5000 rpm, 15min). Subsequently, the concentration of resulted free quercetin solution was calculated by the above regression curve. The formulas used for the computation of EE and drug loading (DL) of QTSL or AQTSL were expressed as follows[22]:

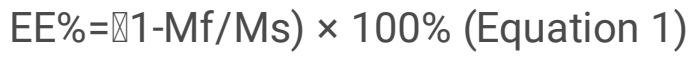

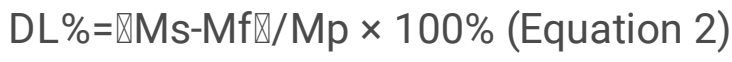


Where Mf, Ms, and Mp represented the mass of free quercetin that unencapsulated in liposomes after ultrafiltration, the total mass of the supplemented quercetin, and the total mass of the ingredients of the prescription for AQTSL respectively.

The in vitro drug release profiles of AQTSL under different temperatures were obtained by the dialysis method as we reported previously [18]. $100 \mathrm{~mL}$ of PBS (pH7.4) was placed in a $250 \mathrm{~mL}$ beaker. After 2 $\mathrm{mL}$ of AQTSL suspension was loaded into a dialysis bag and hung in the beaker containing dialysis buffer, the contents of the beaker were stirred at a speed of $300 \mathrm{rpm}$ by a magnetic stirrer under $37^{\circ} \mathrm{C}$ or $42{ }^{\circ} \mathrm{C}$ to promote drug release. $2 \mathrm{~mL}$ of sample was taken from the beaker at specific time points and the same volume of dialysis buffer was supplemented at the same time. The concentrations of quercetin in the taken samples were determined according to the standard curve of above.

\subsection{Cell proliferation inhibitory effects of AQTSL in vitro}

To assess the targeting inhibitory effects mediated by AS1411 of AQTSL on cancer cells in vitro, HeLa cells were placed in a 96-well culture plates $\left(1 \times 10^{4}\right.$ cells/well) and further cultured for 24 hours $\left(37^{\circ} \mathrm{C}, 5 \%\right.$ $\mathrm{CO}_{2}$ ). Next, QTSL or AQTSL with quercetin final concentration of $50 \mu \mathrm{g} / \mathrm{mL}$ were added into the culture plates, and culture medium was taken as blank control. After $48 \mathrm{~h}$ of treatment, the proliferation inhibition activity was determined by SRB staining method [19]. The absorbance of blank control cells was considered as $100 \%$, cell viabilities of different nanoparticle treatments were calculated according to the following formula[20].

$C V \%=\left(A_{540 \mathrm{~T}} / A_{540 \mathrm{BC}}\right) \times 100 \%$ (Equation 3$)$

Where $\mathrm{CV}$ represented the cell viabilities of treatment group, $A_{540 \mathrm{~T}}$ and $\mathrm{A}_{540} \mathrm{BC}$ were the absorbance values of treatment group cells and the blank control cells at $540 \mathrm{~nm}$.

\subsection{Cytotoxicity of temperature dependent-drug release of AQTSL on HeLa cells}

To explore the thermosensitive release of AQTSL in vitro, we obtained the 30 min quercetin release after incubation under $37^{\circ} \mathrm{C}$ and $42^{\circ} \mathrm{C}$ respectively, followed by an SRB assay to detect the in vitro cytotoxicity of different concentrations of quercetin release from AQTSL on HeLa cells. Briefly, AQTSL was incubated in a water bath at $37{ }^{\circ} \mathrm{C}$ or $42{ }^{\circ} \mathrm{C}$ respectively for $30 \mathrm{~min}$ to release quercetin. After drug releasing, ultrafiltration and centrifugation were applied to separate the released quercetin, and then the released quercetin was diluted to $10 \%, 20 \%, 40 \%, 60 \%$, and $80 \%$ of the initial concentration with DMEM. $200 \mu \mathrm{L}$ HeLa cells were seeded in 96-well culture plates at a density of $5 \times 10^{4}$ cells $/ \mathrm{mL}$ and further cultured for 24 hours $\left(37^{\circ} \mathrm{C}, 5 \% \mathrm{CO}_{2}\right)$. Then $100 \mu \mathrm{L}$ quercetin diluted solution of above was added to each well and an SRB staining assay was conducted as the method of 2.6 .

\subsection{Cell Apoptosis Assay}


The Annexin V-FITC/PI Apoptosis Detection Kit was used to detect the apoptosis inducing ability of different nanoparticles in vitro. After being treated with different nanoparticles, the cells in 6-well plate were washed three times by precooled PBS and then digested by trypsin without EDTA. The collected cells were resuspended with $500 \mu \mathrm{L}$ binding buffer and incubated with annexin V-FITC for 15 min and PI for 5 min respectively in darkness, and then analyzed by flow cytometry (Becton Dickinson, FacsCalibur, USA).

\subsection{Flow cytometry}

To clarify the targeting ability of AQTSL, flow cytometry was conducted further. HeLa cells were seeded in a 6-well plate $\left(1.0 \times 10^{5}\right.$ cells per well) and digested by trypsin after reaching a $90 \%$ fusion rate to $1.5 \mathrm{~mL}$ centrifuge tubes. Then $300 \mu \mathrm{L}$ of FAM-NCQTSL or FAM-AQTSL and $200 \mu \mathrm{L}$ of binding buffer $(4.5 \mathrm{mg} / \mathrm{mL}$

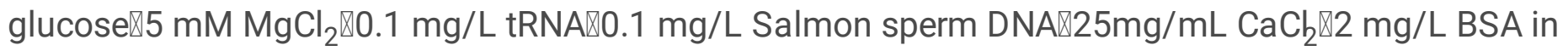
PBS) were added to each tube and incubated at $37^{\circ} \mathrm{C}$ for $1 \mathrm{~h}$. After washing three times using washing buffer $(4.5 \mathrm{mg} / \mathrm{mL}$ glucose, $1 \mathrm{mg} / \mathrm{mL} \mathrm{MgCl}$ in PBS), cells resuspended in PBS were subjected to flow cytometry analysis, and the FL1 detection channel was selected for detection at an excitation wavelength of $488 \mathrm{~nm}$. The affinity between different nanoparticles and different cells was represented by the fluorescence intensity (I). The fluorescence intensity was calculated by the formula as follow.

$\mathrm{I}=\log (\mathrm{x}$-mode $) \times 340($ Equation 4$)$

\subsection{Antitumor activity of AQTSL in vivo}

The density of U14 cells was adjusted to $1 \times 10^{7} / \mathrm{mL}$, and $0.2 \mathrm{~mL}$ cell suspension was injected subcutaneously into the armpit of the left upper limb of each mouse to establish a cervical cancerbearing mouse model. Then the model mice were randomly divided into 9 groups with 6 mice in each group (Day 0): 1) physical saline (Saline), 2) free DOX (DOX, positive control), 3) blank thermosensitive liposomes (BTSL), 4) blank thermosensitive liposomes $\left(+42^{\circ} \mathrm{C}\right.$ heating) $\left.(B T S L+H), 5\right)$ free quercetin (free-Que), 6) quercetin thermosensitive liposomes (QTSL), 7) quercetin thermosensitive liposomes (+ 42 ${ }^{\circ} \mathrm{C}$ heating) (QTSL+H), 8) aptamer-modified quercetin thermosensitive liposomes (AQTSL) and 9) aptamer-modified quercetin thermosensitive liposomes $\left(+42^{\circ} \mathrm{C}\right.$ heating) (AQTSL+H) and the corresponding treatment in each group was carried out according to the experimental design, the doses of different formulae required by each group ( $D O X=5 \mathrm{mg} / \mathrm{kg}$.bw, quercetin= $5 \mathrm{mg} / \mathrm{kg} . \mathrm{bw}$, the quercetin doses in QTSL and AQTSL=5 mg/kg.bw) was based on our preliminary experiment. After $24 \mathrm{~h}$ of inoculation (Day 1), the tail vein injection was conducted every two days for a total of 7 times, and the left upper limb axilla of mice in $42^{\circ} \mathrm{C}$ administration groups were soaked into $42^{\circ} \mathrm{C}$ water bath after tail vein injection $5 \mathrm{~min}$ for $5 \mathrm{~min}$. The groups administrated with Saline, BTSL and free DOX were chosen as the negative control, blank carrier control and the positive control, respectively. Throughout the experiment, the experimental animals were free to eat and water. The bodyweights of mice were weighed every day, and the health indicators such as activities and feeding status were monitored. After the formation of 
solid tumor, the maximum diameters and the minimum diameters of tumors were measured and recorded every two days, and the tumor volumes were calculated according to the following formula.

$\mathrm{V}\left(\mathrm{mm}^{3}\right)=0.5 \times \mathrm{D}_{(\max )} \times \mathrm{D}_{(\min )^{2}}$ (Equation 5$)^{2}$

Where $\mathrm{V}$ represented the volume of the tumor, $\mathrm{D}_{(\max )}$ stands for the maximum diameter and $\mathrm{D}_{(\mathrm{min})}$ stood for the minimum diameter of the measured tumor [21].

On the $16^{\text {th }}$ day of the tumor implantation (Day 15), all the experimental mice were sacrificed under ether anesthesia. The heart, liver, kidney, spleen, thymus and tumor tissues were dissected carefully, weighed accurately, and stained by H.E for pathological observation respectively. The tumor inhibition rate was calculated by the formula as follow.

$\operatorname{TIR}(\%)=(1-\mathrm{Wa} / \mathrm{Wn}) \times 100 \%($ Equation 6$)$

TIR was the tumor inhibition rate, $\mathrm{W}_{\mathrm{a}}$ represented the tumor weight of the different administration group, and $W_{n}$ stood for the tumor weight of the negative control (normal saline) group.

The organ coefficients were expressed by the following formula.

$\mathrm{OC}(\mathrm{mg} / 10 \mathrm{~g})=\mathrm{O}_{\mathrm{W}}(\mathrm{mg}) / \mathrm{B}_{\mathrm{W}}(\mathrm{g}) \times 10($ Equation 7$)$

$O C$ represented the organ coefficient of different organs in mice, $\mathrm{O}_{W}$ and $\mathrm{B}_{\mathrm{W}}$ stood for the organ weight and the body weight of mice respectively.

The blood of experimental animals was collected and the serum was separated for the determination of liver function and renal function indexes (ALT, AST, BUN and CRE) to evaluate the toxic effects of different nanoparticles further.

\subsection{Hemolysis experiment}

Different concentrations of AQTSL $(62.5,125,250$, and $500 \mu \mathrm{g} / \mathrm{mL}$, respectively), physical saline (negative control) and distilled water (positive control) were added to chicken erythrocyte to evaluate the adverse effects of AQTSL. Next, the samples were evenly mixed and incubated at $37^{\circ} \mathrm{C}$ for 30 minutes, further centrifuged ( $5000 \mathrm{rpm}, 5 \mathrm{~min}$ ) and separated to obtain the supernatants. Finally, a UV-Vis spectrophotometer (UV-2550, Shimadzu Ltd., Japan) was applied to determine the absorbance of each sample supernatant at $570 \mathrm{~nm}$ wavelength. The hemolysis rate was calculated according to the following formula.

$H R(\%)=\left[\left(A_{s}-A_{n}\right) /\left(A_{p}-A_{n}\right)\right] \times 100 \%$ (Equation 8)

Where HR stood for the hemolysis rate for different concentrations of AQTSL administrated samples. $A_{s}$, $A_{n}$ and $A_{p}$ represented the absorbances of AQTSL treated samples, negative control (physical saline) 
treated sample and positive control (distilled water) treated samples, respectively.

2.12. Statistical analysis

All the experiment obtained data were expressed as mean $\pm S D$ with three independent experiments. The data analysis was performed using Graphpad Prism 8 software (GraphPad Software Inc., La Jolla, and CA. USA). Multiple comparisons analyzed by variance analysis and considered $p<0.05$ significant.

\section{Results And Discussion}

\subsection{Synthesis and characterization of AQTSL}

AQTSL was prepared by the film hydration method combined with the amide reaction of AS1411 with DSPE. Both QTSL and AQTSL were round spheres with uniform diameters and ideal dispersion characteristic without aggregation and adhesion, with an average particle size of around $100 \mathrm{~nm}$ and 150 $\mathrm{nm}$ respectively (Figure $2 \mathrm{~A}$ and $2 \mathrm{~B}$ ).

Agarose gel electrophoresis, UV spectroscopy, particle size and zeta potential, and mass spectrometry were adopted to further verify the successful modification of AS1411 on the QTSL. Figure 1C showed that compared with AS1411, the electrophoretic mobility of AQTSL was blocked, indicating that AS1411 was successfully coupled to QTSL, resulting in the increased molecular weight and slower migration rate. There were multiple electrophoresis strips for AQTSLs, which might be due to the modification of unequal amounts of AS1411 on the surface of QTSL, which leads to unequal migration distances by electrophoresis. The UV-vis absorption spectra of AQTSL and QTSL were shown in Figure 1D, the appearance of the peak near $260 \mathrm{~nm}$ was also attributed by the successful modification of AS1411 on the QTSL.

Furthermore, the successful modification of AS1411 on the QTSL surface could be further confirmed by changes in particle size and Zeta potential. Figure 1E and 1F showed the particle size of AQTSL was increased to $166.8 \pm 5.92 \mathrm{~nm}$ from $121.5 \pm 4.66 \mathrm{~nm}$ of QTSL, and the Zeta potential of AQTSL was decreased to $-9.1 \pm 0.12 \mathrm{mV}$ from $-7.1 \pm 0.12 \mathrm{mV}$ of QTSL. Finally, the liquid chromatography-mass spectrometry (LC-MS) was adopted to verify the successful decoration of AS1411 on QTSL. Results in Figure $1 \mathrm{G}$ showed that compared with the raw material mixture and QTSL, AQTSL possessed the massto-charge ratio at the 331.4000 bit, which was the fragment ion peak unique to AS1411, further proving the successful linkage of AS1411 to QTSL.

3.2 EE, DL, stability analysis, and drug release profile of AQTSL in vitro

The EE, DL of quercetin within QTSL were found to be $71.5 \pm 0.75 \%$ and $7.24 \pm 0.32 \%$ respectively according to the method of ultracentrifugation combined with UV spectrum analysis. Noteworthy, the aptamer modification on the QTSL showed no influence on the EE $(70.9 \pm 0.81 \%)$ and DL $(6.94 \pm 0.42 \%)$ of AQTSL, which were similar to the non-targeted nanoliposomes. 
DSC was performed to investigate the temperature sensitivity of AQTSL. Figure 2A showed that there was a major phase transition peak near $42{ }^{\circ} \mathrm{C}$ which was due to the presence of MSPC lysolipid in the lipid bilayer. The MSPC lysolipid tends to stabilize the bilayer in the gel phase at temperature lower than $42{ }^{\circ} \mathrm{C}$ and destabilize the bilayer in a disordered structure at temperature higher than $42{ }^{\circ} \mathrm{C}$.

Next, AQTSL was stored at $4^{\circ} \mathrm{C}$ and its solution color, clarity, particle sizes, and Zeta potentials were observed and tested weekly to determine the stability of AQTSL. After AQTSL was stored for four weeks, its solution color kept light yellow-green, clear, and transparent, no agglomeration, and no significant color changes were observed. After 1, 2, 3, and 4 weeks of storage, the particle sizes (Figure 2B), zeta potentials (Figure 2C), and PDI values (Figure 2D) showed no significant changes, which indicated that AQTSL could be stored at $4^{\circ} \mathrm{C}$ for at least four weeks with stable existence.

The in vitro drug release profile showed that the drug release rate increased at both $37^{\circ} \mathrm{C}$ and $42^{\circ} \mathrm{C}$ with the time extension. The cumulative drug release rate of $A Q T S L$ at $37^{\circ} \mathrm{C}$ reached a maximum of $18.0 \pm$ $0.52 \%$ after $20 \mathrm{~min}$ and showed no significant change until $30 \mathrm{~min}$. While, the cumulative drug release increased from $8.1 \pm 0.64 \%$ to $64.0 \pm 0.84 \%$ from 3 to $7 \mathrm{~min}$ at $42^{\circ} \mathrm{C}$, and reached $82.5 \pm 0.92 \%$ by the time of $30 \mathrm{~min}$, indicating that AQTSL achieved a rapid release in a short time (Figure 2E). This result showed that AQTSL was sensitive to the temperature of $42^{\circ} \mathrm{C}$ and showed thermal responsiveness.

3.3. Inhibition effect of AQTSL on cell proliferation and confirmation of tumor targeting of AQTSL in vitro

To verify the thermosensitive release of AQTSL in vitro, we obtained the quercetin release after $30 \mathrm{~min}$ incubation under $37^{\circ} \mathrm{C}$ and $42^{\circ} \mathrm{C}$ respectively, followed by an SRB assay to detect the in vitro cytotoxicity of different concentrations of quercetin release from AQTSL on HeLa cells. As illustrated in Figure 3A, the cell viability decreased little by quercetin release under $37^{\circ} \mathrm{C}$ pre-incubation of AQTSL. While, the cell viability decreased significantly for the treatment by quercetin release under $42^{\circ} \mathrm{C}$ and presented a good correlation between concentration and inhibition effect. We proposed that under $37^{\circ} \mathrm{C}$, quercetin was encapsulated into liposomes and showed a significant slow-release, and exhibited a lower cell inhibition effect than $42^{\circ} \mathrm{C}$, which coincided with the result of the accumulative release profile of above. After the HeLa cells were treated with the release of AQTSL after $42^{\circ} \mathrm{C}$ incubation, the cell survival rates at different concentrations were much lower than that of the treatment incubated under $37^{\circ} \mathrm{C}$, indicating that $42^{\circ} \mathrm{C}$ administration caused the phase transformation of the liposome membrane from a gel state to liquid crystal state, further resulting in the burst release of quercetin from liposomes.

To confirm the increased drug cytotoxicity induced by the enhanced cellular uptake of the nanoparticles due to aptamer conjugation, a time-dependent cytotoxicity assay was conducted. The result in Figure 3B showed AQTSL showed a more rapid and stronger inhibitory effect on HeLa cells proliferation, this result confirmed that aptamer AS1411 played an important role in selectively delivering quercetin and promoting the entry of the nanoparticles by binding with the nucleolin on the surface of the tumor cell membrane. In addition, AS1411 aptamer itself can also act as an aptamer drug and exhibit antiproliferative activity against cancer cells through binding with nucleolin, which might attribute to the proliferation inhibition effect of AQTSL on HeLa cells $[14,15]$. 
To further clarify that the cell growth inhibition effects of AQTSL relied on the apoptosis-inducing pathway, we performed apoptosis experiments. As shown in Figure 3C, the apoptosis rates of BTSL, QTSL, and AQTSL treatment groups were $8.45 \pm 1.34 \%, 13.25 \pm 1.55$, and $21.74 \pm 1.12 \%$, respectively for HeLa cells. Compared with BTSL, both QTSL and AQTSL treatments showed a significant apoptosisinducing effect on Hela cells for both of the nanoparticles (QTSL and AQTSL) containing quercetin. Compared with QTSL, AQTSL treatment cells showed a higher apoptotic rate, further suggesting that the surface modification of AS1411 on the quercetin temperature sensitive liposomes increased the targeting of the nanoparticles and induced more apoptosis of tumor cells.

Next, an SRB assay was performed to evaluate the cytotoxicity of AQTSL and NCQTSL against 293T cells and HeLa cells respectively to verify the tumor-targeting effects of AS1411 decoration on the liposomes. NCQTSL and AQTSL were incubated with 293T cells or HeLa cells respectively and the cell toxicity results were shown in Figure 3D and 3E. The viability of 293T cells did not decrease obviously after treatment with AQTSL compared with NCQTSL incubation ( $p>0.05)$, which illustrated that the delivery system possessed well biocompatibility to normal cells. While, the viability of HeLa cells decreased significantly after incubation with AQTSL compared with NCQTSL $(p<0.01)$, which indicated that AS1411 decoration improved the tumor tissue active targeting and endocytosis of the nanoliposomes through the binding of AS1411 with nucleolin on the membranes of tumor cells.

Next, in order to further confirm that AS1411 mediated enhanced cellular uptake of nanoparticles by tumor cells, the uptake experiments of FAM-NCQTSL and FAM-AQTSL by tumoral HeLa cells and normal 293T cells were carried out by flow cytometry. After 293T cells and Hela cells were incubated with the above two kinds of nanoparticles respectively, the fluorescence intensity of the cells was detected by flow cytometry. As shown in Fig. 3F a, b and c, the fluorescent intensity of HeLa cells incubated with FAMAQTSL strengthened significantly ( $~ 5$ times) than that of the non-AS1411 functionalized nanoliposomes (FAM-NCQTSL), illustrating the aptamer AS1411 modification increased AQTSL nanoparticles internalization of tumor cells by binding to the nucleolin on the surface of tumor cells. While, there was no significant difference between the fluorescence intensity of 293T cells after incubated with FAM-AQTSL and FAM-NCQTSL (Figure 3E d, e, f), indicating that the internalization of AS1411 modified nanoparticles was not enhanced significantly for the normal cells. The affinity between different nanoparticles and different cells was represented by the fluorescence intensity $(I)$ in Figure 3G, showing more clearly that the affinity between AQTSL and tumor cells was much stronger than that between AQTSL and normal cells.

\subsection{Anti-tumor effects of AQTSL in vivo}

The in vivo antitumor properties of AQTSL were evaluated using cervical cancer harboring mice models. Firstly, the model mice were grouped into 9 treatment randomly, administration once on alternate days, and 15 days for a therapy course (Figure 4A). The body weight of mice in all the 9 groups above remained at a similar growth, manifesting the treatment of each group caused no significant systemic toxicity (Figure 4B). As illustrated in Figure 4C, D, E, and F, BTSL demonstrated no tumor growth difference in comparison to the Saline treatment, indicating the blank vector possessed no inhibition 
effect on tumor. Tumors in both BTSL $+\mathrm{H}$ and free Que treatment groups showed smaller sized tumors compared with those in saline or BTSL groups, owing to tumor cells are more sensitive to $42{ }^{\circ} \mathrm{C}$ temperature than the normal tissues, and heat shock protein was induced by high temperature and resulting in the tumor cell apoptosis further [22-24]. Free quercetin can also induce tumor cell apoptosis, which has been reported previously $[25,26]$. But the tumor inhibition effects of these two administrations were rather limited. Compared with free Que, QTSL exhibited a stronger tumor inhibition effect, for the quercetin encapsulation in liposomes and surface modification by PEG-2000, which improved the targeting effect and long-term circulation of quercetin. Furthermore, QTSL combined with thermotherapy increased tumor growth inhibition effects. It is noteworthy that AQTSL combined with thermotherapy possessed the most significant tumor inhibition effect compared with all other treatment groups, through EPR effect induced passive targeting effect, the binding of AS1411 with nucleolin resulting active targeting effect and endocytosis promoting effect, PEG modification generated long term circulation, thermal sensitive drug release improving local quercetin concentration in tumor tissues and thermotherapy.

Furthermore, $\mathrm{H} \& \mathrm{E}$ staining examination in $4 \mathrm{G}$ shows that tumor tissue treated with $\mathrm{AQTSL}+\mathrm{H}$ exhibits severe vacuolization, and typical apoptosis and necrosis characteristics, indicating that the degree of differentiation was significantly higher and the degree of malignancy was significantly lower than those of the other treatment groups.

\subsection{Biocompatibility evaluation of AQTSL in vivo}

To further clarify whether the treatments produced toxic side effects on the mice, the organ index measurement, the biochemistry assays (ALT, AST, BUN, and Cre) of the mice serum, the pathological analysis of main organs (liver and kidney), and hemolysis experiment were conducted. The result in Fig. $5 \mathrm{~A}$ showed that there were no significant differences between treatment groups and the Normal saline group in all the main organ coefficients, which further demonstrated that all these treatments had no toxic or side effects on the main organs. The results of the hepatic and renal function indicator showed that there were no significant differences between the normal control group and the other treatment groups (Figure 5B, 5C, 5D, and 5E). Accordingly, the liver tissues treated with AQTSL+H showed that the hepatic cord was clear and no hepatocyte lesion was observed, which were similar to the liver microstructure of normal mice. The kidney tissues of AQTSL+H treatment mice, which were similar to the kidney micro-structure of normal mice showed that the structure of the renal tubule and glomerulus was intact, and the epithelial structure of the renal tubule was normal without degeneration (Figure 5F). Finally, we conducted hemolysis test to evaluate the impact of AQTSL on blood tissue, result in Figure 5G manifested that the hemolysis rates caused by different concentrations of AQTSL were all less than $5 \%$, indicating that AQTSL could entered the circulatory system through intravenous injection, and caused no damage to blood cells during the process of circulation, exhibited an ideal biosafety. The outstanding therapeutic effects of AQTSL+H in vivo were coincided with the in vitro cytotoxicity results and confirmed that thermal-controlled release of quercetin, passive targeting and active targeting transportation, together with chemotherapy and thermotherapy were effective for the therapy of tumor. 
Noteworthy, during the in vivo experiment, we applied a $42^{\circ} \mathrm{C}$ water bath for the exogenous heating treatment, which will limit the practical application of our drug carriers. Our present experiment focuses on the thermosensitive liposomes' response to thermal. In the later stages, we will use the hydrophilic cavity of these liposomes to encapsulate certain photothermal conversion materials, such as polydopamine, graphene oxide et al., and use their photothermal conversion to transform near-infrared light to endogenous heat to achieve the controlled release of drugs. This series of research work is being conducted in our lab.

\section{Conclusion}

In this work, we firstly prepared DSPE-PEG2000-AS1411 by coupling $-\mathrm{NH}_{2}$ in $\mathrm{NH} 2-\mathrm{AS} 1411$ and -COOH in DSPE-PEG2000-COOH, and successfully prepared aptamer-modified quercetin thermosensitive liposomes (AQTSL) using quercetin as a model drug, and investigated the in vitro and in vivo anti-tumor activity of AQTSL using MTT assay, cell proliferation assay, flow cytometry and mouse bearing cervical cancer U14 models. This targeted anti-tumor drug carrier combines the merits of high-loading yield, sustained drug release, long-term circulation in the body of PEGylated liposomes, passive targeting of 100-200 nm nanoparticles, active targeting, and improved internalization effect offered by aptamer AS1411, and quercetin releasing controlled by thermal-response. The excellent biocompatibility, cancer cell recognition ability, efficient tunable drug release will provide new horizons in clinical cancer therapy and cancer pharmaceutical development.

\section{Declarations}

Availability of data and materials

The datasets used and/or analysed during the current study are available from the corresponding author on reasonable request.

Competing interests

The authors declare that they have no competing interests.

Funding

This work was supported by the National Natural Science Foundation of China (No. 62071413), the Hebei Natural Science Foundation (No. C2019203556, F2020203056), Hebei education department key project (No. ZD2020147), and Hebei Province Key Research and Development Projects in China (No. 19272401D).

Authors' contributions

JL designed the whole experiment and was a major contributor in writing the manuscript. YG performed the synthesis and characterization of drug delivery system. SL and JC completed the animal experiment. 
QZ performed the cell experiment in vitro. ZL analyzed the experimental data. KL and JW provided the funding and resources. MS and HC supervised the whole experimental process. All authors read and approved the final manuscript.

Acknowledgements

We would like to thank Prof. Xin Su (Beijing University of Chemical Technology, China) for the writing guidance of this paper and Prof. Zhijie Chang (Tsinghua University, China) for the cell lines gifted.

\section{References}

1. Corti, A., et al., Targeted drug delivery and penetration into solid tumors. Med Res Rev, 2012. 32(5): p. 1078-91.

2. Qiu, L., et al., Self-assembled pH-responsive hyaluronic acid-g-poly((L)-histidine) copolymer micelles for targeted intracellular delivery of doxorubicin. Acta Biomater, 2014. 10(5): p. 2024-35.

3. Malam, Y., M. Loizidou, and A.M. Seifalian, Liposomes and nanoparticles: nanosized vehicles for drug delivery in cancer. Trends Pharmacol Sci, 2009. 30(11): p. 592-9.

4. Sun, B. and S.S. Feng, Trastuzumab-functionalized nanoparticles of biodegradable copolymers for targeted delivery of docetaxel. Nanomedicine (Lond), 2009. 4(4): p. 431-45.

5. Abnous, K., et al., A novel chemotherapy drug-free delivery system composed of three therapeutic aptamers for the treatment of prostate and breast cancers in vitro and in vivo. Nanomedicine, 2017. 13(6): p. 1933-1940.

6. Dam, D.H., et al., Direct observation of nanoparticle-cancer cell nucleus interactions. ACS Nano, 2012. 6(4): p. 3318-26.

7. He, L., et al., Nucleolin-targeted selenium nanocomposites with enhanced theranostic efficacy to antagonize glioblastoma. J Mater Chem B, 2017. 5(16): p. 3024-3034.

8. Taghavi, S., et al., Polyethylenimine-functionalized carbon nanotubes tagged with AS1411 aptamer for combination gene and drug delivery into human gastric cancer cells. Int J Pharm, 2017. 516(1-2): p. 301-312.

9. Luo, Z., et al., Precise glioblastoma targeting by AS1411 aptamer-functionalized poly (I-gammaglutamylg/utamine)-paclitaxel nanoconjugates. J Colloid Interface Sci, 2017. 490: p. 783-796.

10. Cho, Y., et al., Modified AS1411 Aptamer Suppresses Hepatocellular Carcinoma by Up-Regulating Galectin-14. PLoS One, 2016. 11(8): p. e0160822.

11. Bates, P.J., et al., G-quadruplex oligonucleotide AS1411 as a cancer-targeting agent: Uses and mechanisms. Biochim Biophys Acta Gen Subj, 2017. 1861(5 Pt B): p. 1414-1428.

12. Lopes-Nunes, J., et al., Aptamer-Functionalized Gold Nanoparticles for Drug Delivery to Gynecological Carcinoma Cells. Cancers (Basel), 2021. 13(16).

13. Carvalho, J., et al., Aptamer-guided acridine derivatives for cervical cancer. Org Biomol Chem, 2019. 17(11): p. 2992-3002. 
14. Girvan, A.C., et al., AGRO100 inhibits activation of nuclear factor-kappaB (NF-kappaB) by forming a complex with NF-kappaB essential modulator (NEMO) and nucleolin. Mol Cancer Ther, 2006. 5(7): p. $1790-9$.

15. Soundararajan, S., et al., The nucleolin targeting aptamer AS1411 destabilizes Bcl-2 messenger RNA in human breast cancer cells. Cancer Res, 2008. 68(7): p. 2358-65.

16. Chen, C., et al., Stimuli-responsive controlled-release system using quadruplex DNA-capped silica nanocontainers. Nucleic Acids Res, 2011. 39(4): p. 1638-44.

17. Pradhan, P., et al., Targeted temperature sensitive magnetic liposomes for thermo-chemotherapy. J Control Release, 2010. 142(1): p. 108-21.

18. Li, J., et al., Antitumor activity and safety evaluation of nanaparticle-based delivery of quercetin through intravenous administration in mice. Mater Sci Eng C Mater Biol Appl, 2017. 77: p. 803-810.

19. Zhang, Y., et al., Targeting therapy with mitosomal daunorubicin plus amlodipine has the potential to circumvent intrinsic resistant breast cancer. Mol Pharm, 2011. 8(1): p. 162-75.

20. Zhou, J., et al., The anticancer efficacy of paclitaxel liposomes modified with mitochondrial targeting conjugate in resistant lung cancer. Biomaterials, 2013. 34(14): p. 3626-38.

21. Velicu, S., et al., Cross-priming of $T$ cells to intracranial tumor antigens elicits an immune response that fails in the effector phase but can be augmented with local immunotherapy. J Neuroimmunol, 2006. 174(1-2): p. 74-81.

22. Lei, M., et al., A dual $\mathrm{pH} /$ thermal responsive nanocarrier for combined chemo-thermotherapy based on a copper-doxorubicin complex and gold nanorods. Nanoscale, 2015. 7(38): p. 15999-6011.

23. Ebrahimi, A., et al., Comparison of anticancer effect of Pleurotus ostreatus extract with doxorubicin hydrochloride alone and plus thermotherapy on erythroleukemia cell line. J Complement Integr Med, 2017. 15(2).

24. Paiella, S., et al., Laser Treatment of Pancreatic Cancer with Immunostimulating Interstitial Laser Thermotherapy Protocol: Safety and Feasibility Results From Two Phase 2a Studies. J Surg Res, 2021. 259: p. 1-7.

25. Czerwonka, A., et al., Anticancer effects of sodium and potassium quercetin-5'-sulfonates through inhibition of proliferation, induction of apoptosis, and cell cycle arrest in the HT-29 human adenocarcinoma cell line. Bioorg Chem, 2020. 94: p. 103426.

26. Davoodvandi, A., et al., Quercetin as an anticancer agent: Focus on esophageal cancer. J Food Biochem, 2020. 44(9): p. e13374.

\section{Scheme}

Scheme 1 is available in the Supplemental Files section

\section{Figures}


A

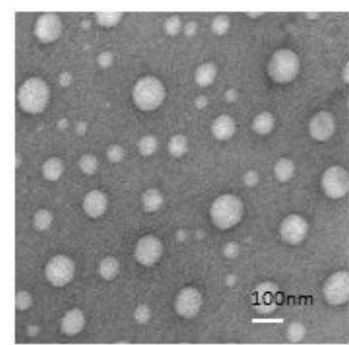

C

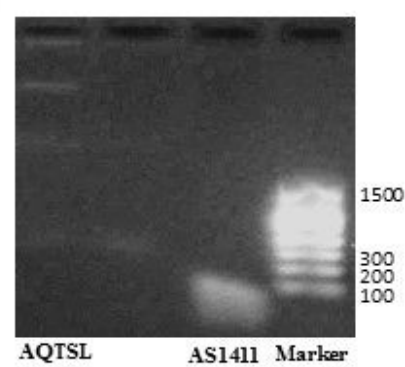

E

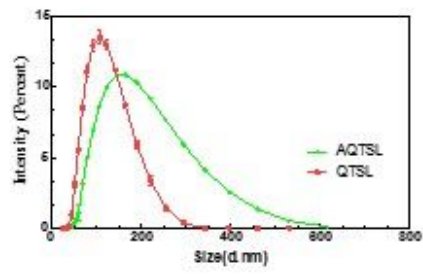

B

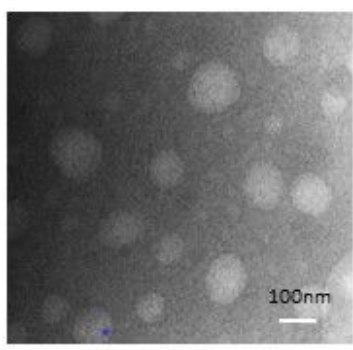

D

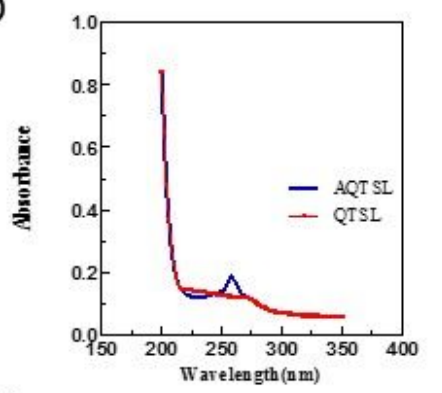

F

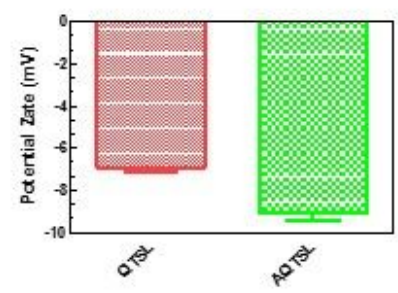

G

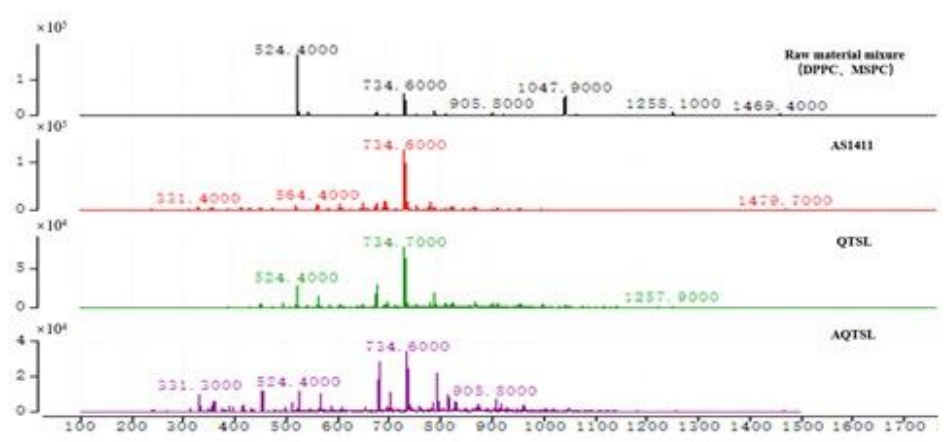

\section{Figure 1}

Characterization of aptamer-functionalized quercetin thermosensitive liposomes (AQTSL). A. TEM image of quercetin thermosensitive liposomes (QTSL). B. TEM image of AQTSL. C. Agarose gel electrophoresis result of AS1411 and AQTSL. D. UV-vis absorption spectra of QTSL and AQTSL. E. Particle size distributions of QTSL and AQTSL. F. Zeta potentials of QTSL and AQTSL. Values represented were 
means $\pm S D(n=3)$. G. liquid chromatography mass spectrometry analysis of raw material mixture, AS1411, QTSL and AQTSL.

A

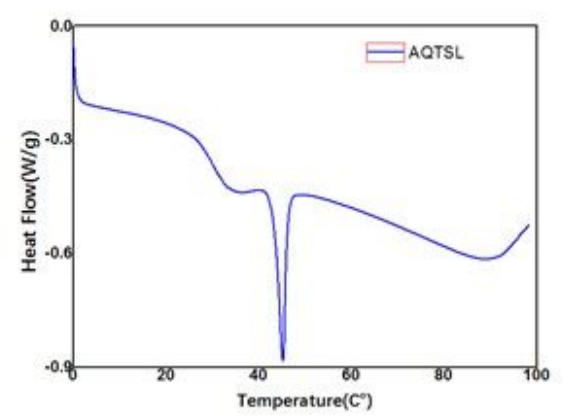

B

C

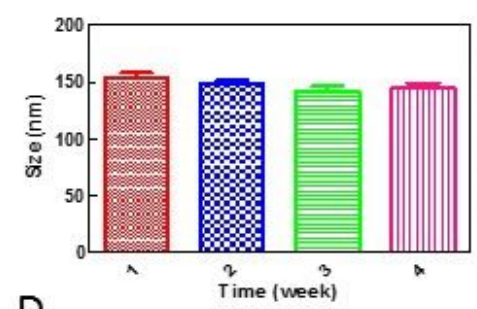

D

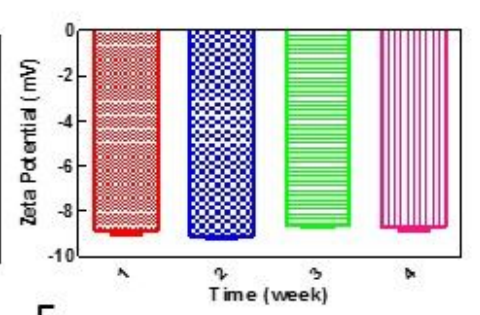

$\mathrm{E}$
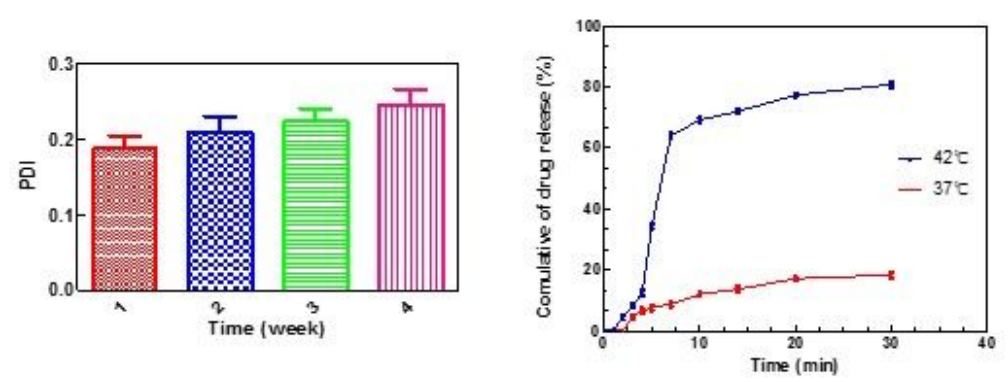

Figure 2

Phase transition temperature, stability analysis and drug release profile of AQTSL in vitro. A. DSC of AQTSL. B. Particle size, C. Zeta potential, and D. PDI changes of AQTSL after 1, 2, 3 and 4 weeks of 
storage at $4{ }^{\circ} \mathrm{C}$. E. Drug release profile of AQTSL under $37^{\circ} \mathrm{C}$ and $42{ }^{\circ} \mathrm{C}$ respectively. Values represented were means $\pm S D(n=3)$.

A

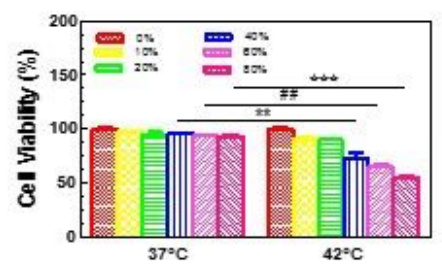

B

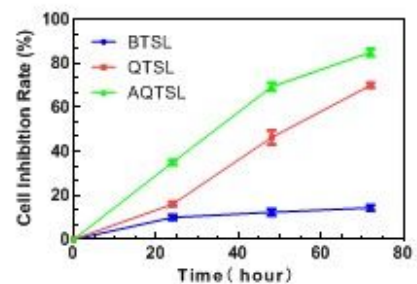

$\mathrm{C}$
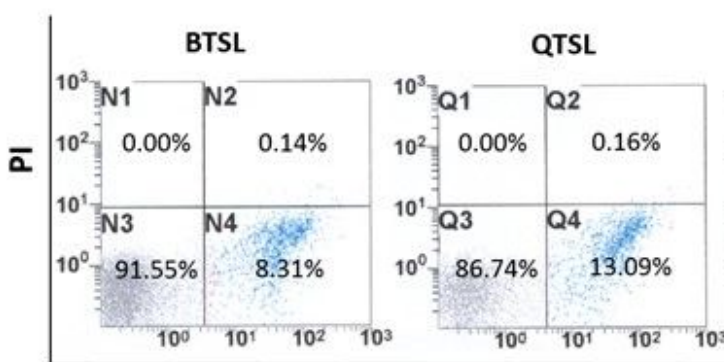

AQTSL

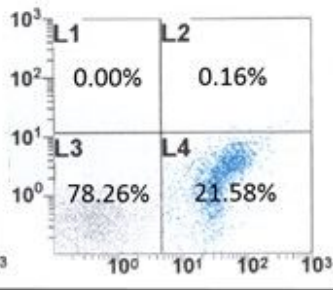

FITC $E$

D

$\mathrm{F}$

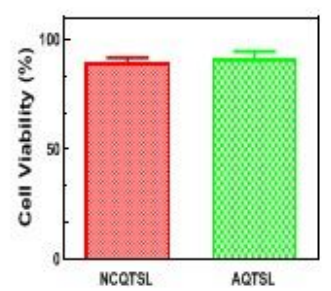

\section{E}
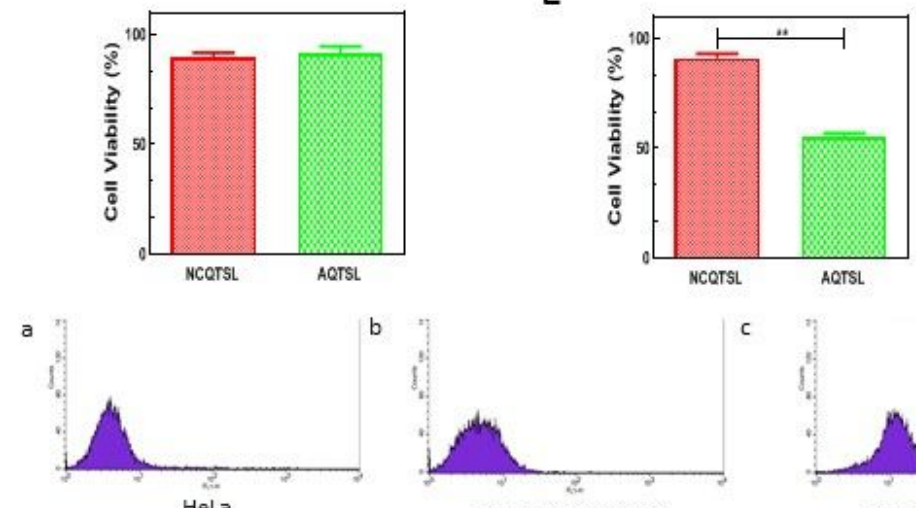

b

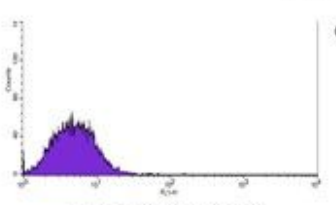

HeLa+FAM-NCQTSL

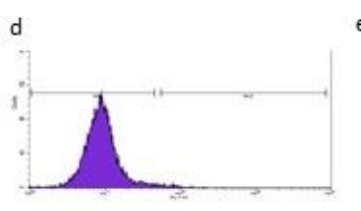

293T

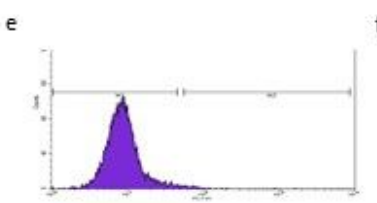

293T+FAM-NCQTSL c

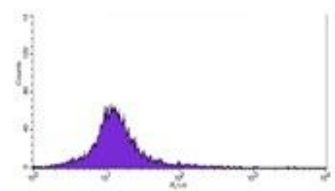

HeLa+FAM-AQTSL

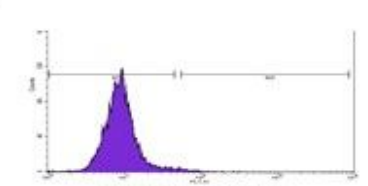

293T+FAM-AQTSL

G

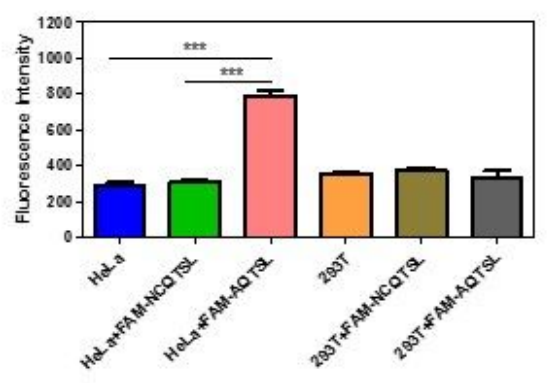

Figure 3

Inhibition effect and tumor targeting activity of AQTSL in vitro. A. MTT assay of quercetin release of 37 ${ }^{\circ} \mathrm{C}$ and $42^{\circ} \mathrm{C}$ incubation on HeLa cells. B. Time dependent cytotoxicity assay of blank thermosensitive 
liposomes (BTSL), QTSL and AQTSL. C. Apoptosis in HeLa cells after incubation with BTSL, QTSL and AQTSL was detected via Annexin V-FITC/PI staining and flow cytometry analysis. Data are expressed as the mean \pm S.D. $(n=3)$. D. Cytotoxicity of NCQTSL and AQTSL on 293T cells. E. Cytotoxicity of NCQTSL and AQTSL on HeLa cells. Values represented were means $\pm S D(n=3)$ and $* * p<0.01$ in comparison with the NCQTSL treatment group. F. Flowcytometry analysis of FAM-NCQTSL and FAM-AQTSL in normal 293T cells and tumoral HeLa cells. G. The affinity between different nanoparticles and different cells was represented by the fluorescence intensity (I). The formula used for computation is expressed in the following manner: $I=\log (x-$ mode $) \times 340$. Data are expressed as the mean \pm S.D. $(n=3) .{ }^{\star \star} p<0.01$ in comparison with the affinity between FAM-AQTSL and HeLa cells. 
A

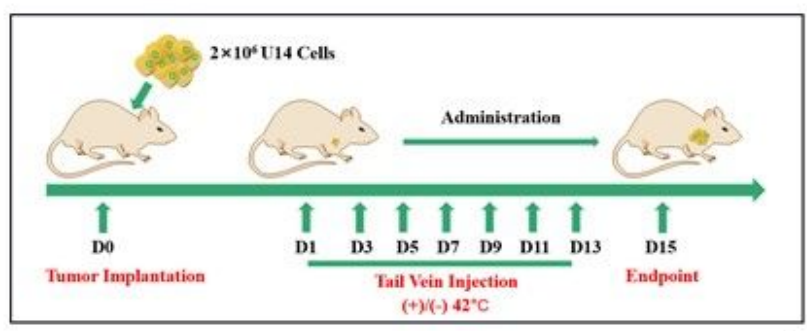

C

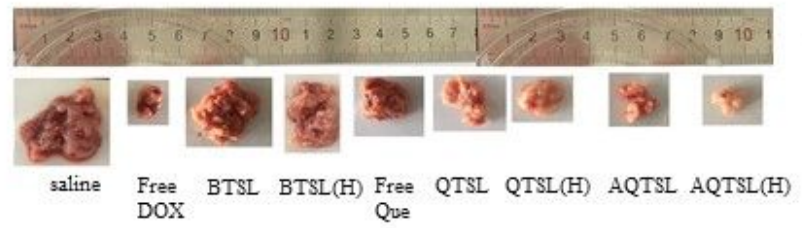

E

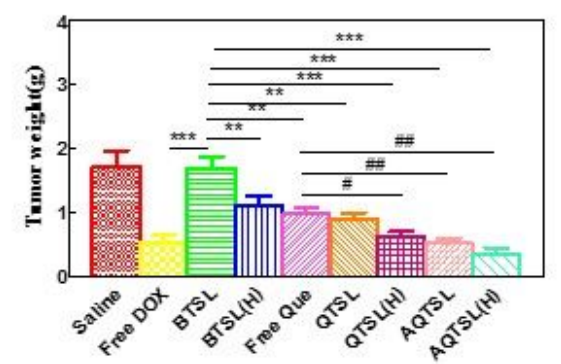

B

\section{D}
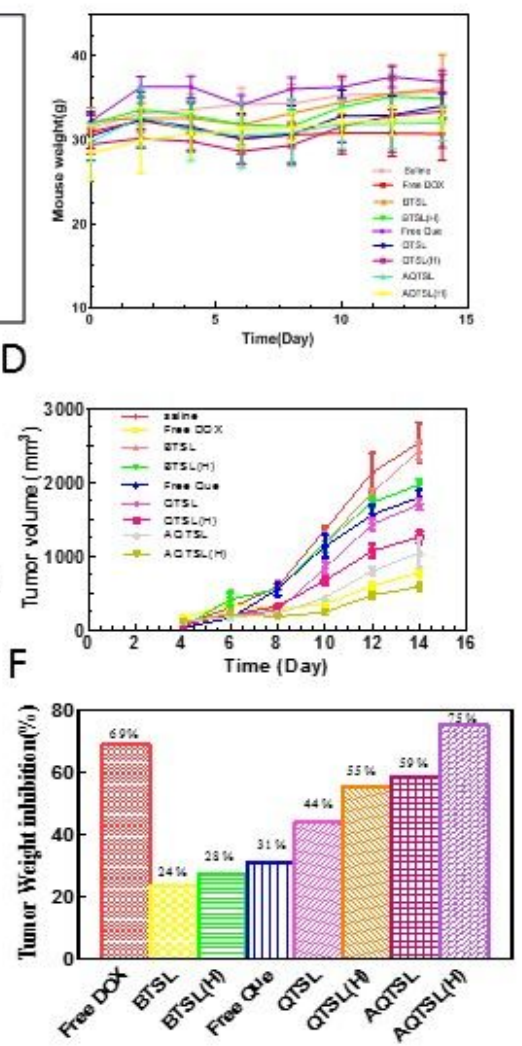

G

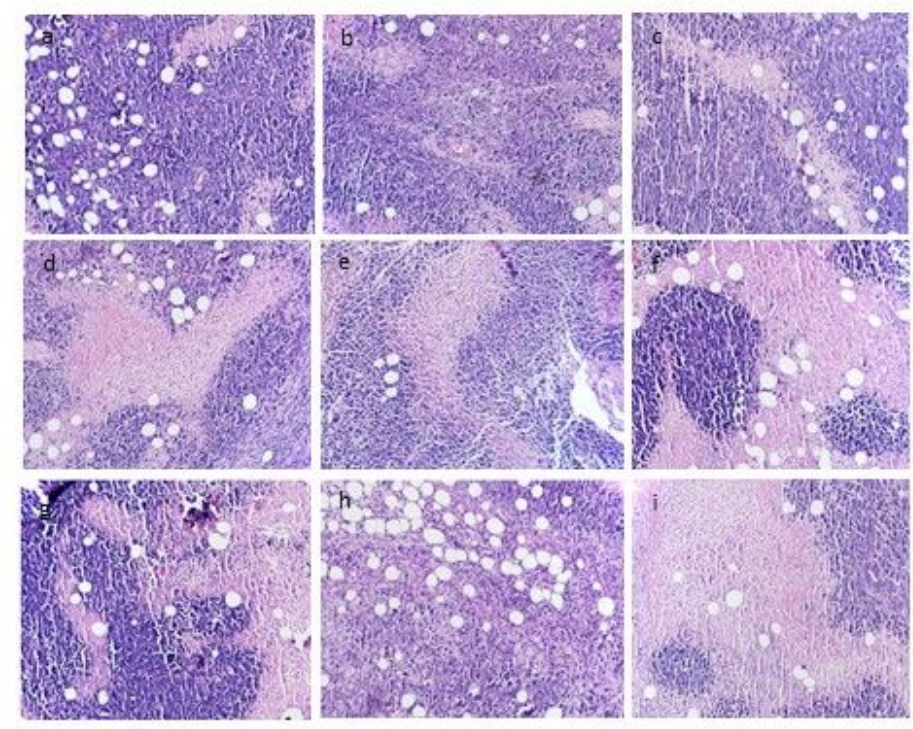

\section{Figure 4}

Anti-tumor effects of AQTSL in vivo. A. Schematic diagram of therapy course on cervical cancer-bearing mice. B. Bodyweight change profiles of mice models after different administration. Values expressed as means $\pm S D(n=6)$. C. Photos of tumors obtained from different groups on the $15^{\text {th }}$ day. D. Tumor volume curves of different treatment groups against time $(n=6)$. E. Quantitative analysis of the tumor weights in different treatment mice on the $15^{\text {th }}$ day after U14 cells inoculation. Values expressed as means \pm SD 
$(\mathrm{n}=6),{ }^{\star} * x<0.01$ and ${ }^{\star \star \star} p<0.001$ compared with BTSL treatment group, $\# p<0.05$ and $\# \# p<0.01$ compared with free quercetin treatment group. F. Tumor growth inhibition rates of different treatment group. G.

Representative H.E staining images of tumor tissues obtained from different treatment group mice. The meaning of $a-i$ is presented as follow treatment, a. Saline; $b$. free DOX; c. BTSL; $d$. BTSL+H; e. free-Que; f. QTSL; g. QTSL+H; h. AQTSL; i. AQTSL+H.

A
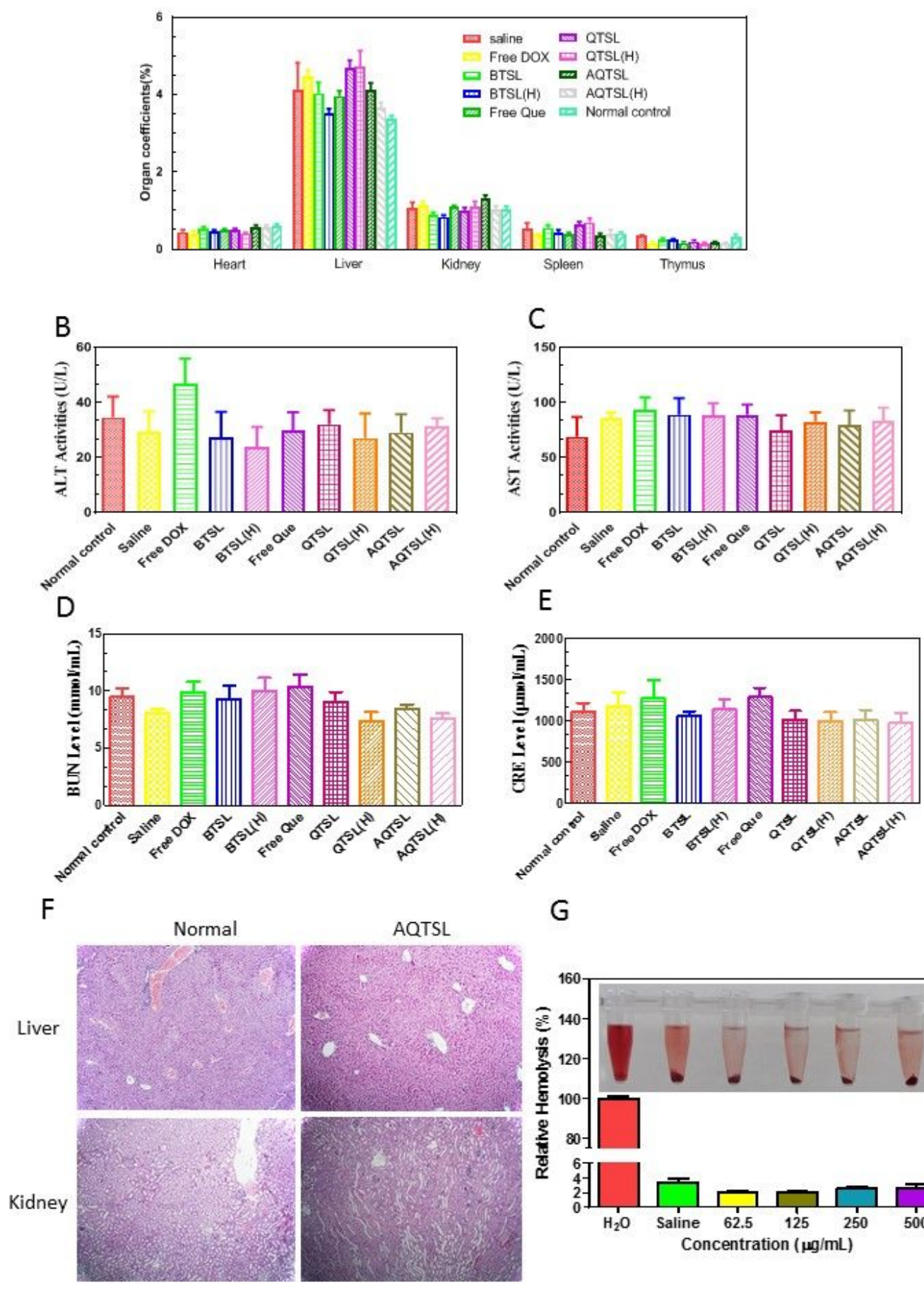

G

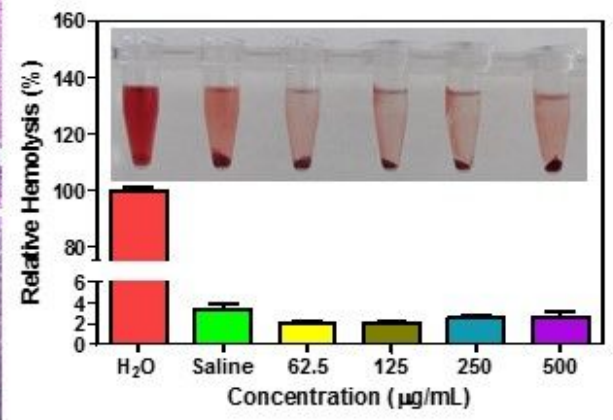

Figure 5 
Biosafety evaluation of AQTSL. A. Organ indexes, B Alanine transaminase activities, C. Aspartate aminotransferase activities, D. Blood urea nitrogen levels and $\mathrm{E}$. Creatinine levels in tumor bearing mice after various treatment. Data are shown in mean $\pm S D(n=6)$. F. The H\&E staining of livers and kidneys in $\mathrm{AQTSL}+\mathrm{H}$ treatment group and the liver and kidney in normal mice were used as the negative control. $\mathrm{H}$. Hemolytic assay of AQTSL in vitro. Values represented were means $\pm S D$. $(n=6)$.

\section{Supplementary Files}

This is a list of supplementary files associated with this preprint. Click to download.

- Scheme1.jpg 